Paidéia, 2005, 15(30), 131-139

\title{
AUTO CONCEITO E IMAGEM CORPORAL EM CRIANÇAS OBESAS ${ }^{1}$
}

\author{
Ana Maria Pimenta Carvalho ${ }^{2}$ \\ Caroline Cataneo \\ Elizângela Moreira Careta Galindo \\ Carolina Tomain Malfará \\ EERP - Universidade de São Paulo
}

\begin{abstract}
Resumo: Este trabalho teve como objetivo descrever o que pensam crianças obesas sobre seus corpos. Para tanto o delineamento metodológico incluiu um grupo de obesos e outro de não obesos. As crianças responderam a dois instrumentos que tocam nessas questões de forma sistematizada - Escala Piers Harris de Auto-Conceito e Eating Behviours and Body Image Test. O grupo de crianças obesas era composto por 14 meninas e 13 meninos, com idades entre 10 a 12 anos e índice de massa corporal variando de P85 a P95 ou acima; o de não obesas era composto por 15 meninas e 12 meninos, com a mesma faixa etária e índice de massa corporal entre $\mathrm{P} 5$ a $\mathrm{P}<85$. Os resultados mostraram que as crianças obesas estão mais insatisfeitas com seu corpo e aparência. Entretanto, elas identificam em si características físicas positivas e outros atributos pessoais. Embora menos preocupadas, as crianças não obesas também manifestam insatisfação, possivelmente devido às pressões culturais sobre a aparência física.
\end{abstract}

Palavras-chave: obesidade;crianças; auto-conceito; imagem corporal.

\section{SELF CONCEPT AND BODY IMAGE IN OBESE CHILDREN}

\begin{abstract}
This study aimed to describe what obese children think about their bodies. The methodological design included two groups - obese and non obese children. The children were asked to answer two instruments that offer a systemized treatment of these questions - the Piers-Harris Self Concept Scale and the Eating Behaviors and Body Image Test. The obese group consisted of 14 girls and 13 boys between 10 and 12 years old, whose body mass index ranged from P85 to P95 or higher. The non obese group consisted of 15 girls and 12 boys in the same age range, whose body mass index ranged from $\mathrm{P} 5$ and $\mathrm{P}<85$. The results show that obese children are more dissatisfied about their body and appearance. However, they also identify positive physical characteristics and other personal attributes in themselves. Although less worried, non obese children also demonstrate dissatisfaction. This may be due to cultural pressure with respect to physical appearance.
\end{abstract}

Key-words: obesity; children; self concept; body image.

\section{Introdução}

Estudos iniciais sobre percepção e satisfação corporal, usando a terminologia de Sheldon para caracterização de tipos físicos, mostraram que meninos e meninas pré escolares atribuiam características

\footnotetext{
${ }^{1}$ Artigo recebido para publicação em 18/03/2004; aceito em 28/01/ 2005

${ }^{2}$ Endereço para correspondência: Ana Maria Pimenta Carvalho, Departamento de Enfermagem Psiquiátrica e Ciências Humanas, Escola de Enfermagem de Ribeirão Preto/USP, Av. Bandeirantes 3900, CEP: 14040-902, Ribeirão Preto/SP, E-mail: anacar@eerp.usp.br
}

positivas como, ser gentil, ter muitos amigos, ser feliz e educado a tipos físicos mesomórficos. As características negativas como, ser brigão, chato, preguiçoso e superficial são mais atribuídas a tipos físicos endomórficos. Estes achados sugerem uma atitude de rejeição a crianças endomórficas, especialmente as do sexo masculino (Lerner \& Korn, 1972; Kirkpatrick \& Sanders, 1978).

Stager e Burke (1982) encontraram em crianças e adolescentes de 9 a 16 anos, identificadas como endomórficas, independente da relação real entre peso e altura, baixa auto-estima e uma associação entre 


\section{Ana Maria Pimenta Carvalho}

seu tipo físico e características pessoais mais negativas que aquelas atribuídas a indivíduos com tipos físicos ectomórficos ou mesomórficos. Na interpretação dos autores, tais resultados sugerem a emergência de auto-percepções com relação ao tipo físico que afetam o senso de eu e a satisfação com o próprio corpo. A maior satisfação encontra-se mais associada a um tipo físico mais magro.

Paxton, Wertheim, Gibbons, Szmukler, Hillier e Petrovich (1991) encontraram, entre adolescentes australianos, especialmente os do sexo feminino, preocupação com o controle de peso. Além disso, eles tendiam a atribuir à magreza características como felicidade e inteligência.

Embora o começo da puberdade aumente o desejo de ser mais magro, há evidências de que no período pré puberal as crianças também se preocupem com seus corpos e busquem perder peso (Lawrence, 1991; Thelen, Powel, Lawrence \& Kuhnert, 1992).

Tais estudos mostram que o tipo físico está sujeito a associação com traços de personalidade, quando avaliado pelo outro; com satisfação e felicidade, quando avaliado pelo próprio indivíduo.

Um dos fatores que mobiliza o indivíduo com excesso de peso (endomórfico) a buscar ajuda para o problema diz respeito ao incômodo com sua imagem corporal que pode vir a interferir com seu senso de auto-estima. Especialmente num contexto em que o corpo magro, esbelto, é valorizado e as roupas da moda são confeccionadas em tamanhos pequenos, este incômodo parece ser maior e vivenciado com sentimentos de raiva, angústia, culpa. Associadas a estes, encontram-se preocupações com a saúde, visto que, tem sido amplamente divulgado, nas diversas modalidades da imprensa, o crescente aumento da obesidade entre a população em geral e, em especial, entre crianças e adolescentes e os riscos de se desenvolver doenças como hipertensão, diabetes tipo II, além de problemas na coluna e articulações, sobretudo de membros inferiores.

Em decorrência disto adultos, pais e filhos têm buscado ajuda, inicialmente médica, para solucionar o problema. Entretanto esta solução não é simples e requer mudanças em hábitos familiares e pessoais muitas vezes arraigados. A literatura vem mostrando que os procedimentos mais eficazes para lidar com a obesidade são aqueles que envolvem o trabalho de equipes multiprofissionais. Assim a compreensão sobre os múltiplos aspectos envolvidos com o fenômeno da obesidade faz-se necessária.

No que tange ao envolvimento de aspectos psicológicos e aqui faremos o recorte para a obesidade infantil, à semelhança do que se faz com a obesidade no adulto, tem-se buscado encontrar ligações entre traços de personalidade e também sinais de sofrimento psicológico expressos por alterações emocionais e comportamentais. Em relação aos adultos, os achados são inconclusivos levando a pensar que não há um padrão de personalidade do indivíduo obeso. Em crianças, têm sido identificadas alterações comportamentais, sinais de sofrimento psíquico e baixo auto-conceito. (Azevedo, 1996; Braet, Mervielde e Vandereycken, 1997). A partir da avaliação psicológica utilizando-se o Teste do Desenho da Figura Humana, com as crianças e o Questionário de Morbidade Psiquiátrica Infantil, respondido por seus pais, Azevedo (1996) encontrou que entre o grupo de crianças obesas achavam-se presentes mais sinais emocionais, expressos pelos desenhos como braços curtos e relatos de problemas comportamentais, tais como retraimento, insegurança.

Utilizando a avaliação de auto-conceito e a avaliação comportamental da criança, fornecida por seus pais, Braet, Mervielde e Vandereycken (1997) compararam três grupos de crianças de 9 a 12 anos sendo 92 obesas em tratamento, 47 obesas que não estavam em tratamento e 150 não obesas. Encontraram diferenças significativas entre os três grupos, mostrando que as crianças que estavam em tratamento apresentaram um auto-conceito mais negativo e suas mães relataram mais problemas comportamentais.

Focalizando, de modo mais específico, as auto-percepções em crianças que buscaram atendimento para o problema da obesidade, Dechen, Cano e Ribeiro (2000) identificaram verbalizações de descontentamento com o excesso de peso e sentimentos de menos valia, devido a chacotas sofridas por companheiros de escola.

Galindo e cols. (2002) identificaram em um grupo de crianças obesas, em inicio de tratamento, manifestações positivas sobre sua aparência física e, ao lado de sinais de descontentamento. 
Resultados divergentes como estes parecem dever-se à forma de abordagem aos participantes dos diferentes estudos e ao instrumento de coleta de dados. Entrevistas foram utilizadas no estudo de Dechen e cols. e uma escala de avaliação de auto-conceito foi utilizada no estudo de Galindo e cols.

Programas de tratamento para a obesidade infantil têm enfatizado quão difícil é conseguir minimizar esta condição. Nem todos os que buscam atendimento têm sucesso mantendo ou reduzindo o índice de massa corporal. Sabe-se, ainda, que o envolvimento da família é de extrema importância, pois são os pais que arranjam o ambiente da criança podendo facilitar ou dificultar o controle da ingestão de alimentos menos calóricos. Em geral são os pais que oferecem à criança os alimentos, diretamente, por meio do preparo de refeições ou permitindo sua compra pela própria criança.

A despeito do envolvimento de fatores como os biológicos e ambientais na determinação da obesidade é importante ressaltar que os aspectos emocionais e comportamentais acham-se associados e quando se pensa em tratamento há que se considerar o modo como o indivíduo chega ao buscar ajuda. Quem busca ajuda? Os pais ou a própria criança? E, ainda que se esteja falando de crianças, não se pode deixar de mencionar as auto percepções como fatores importantes na regulação do comportamento.

No âmbito da teoria cognitivo-social os comportamentos são determinados por fatores ambientais e pessoais. No âmbito pessoal entram os mecanismos de auto-regulação e dentre eles têm um papel fundamental os mecanismos auto-avaliativos, como auto-estima e auto-conceito, referindo-se à avaliação que o indivíduo faz de si, em diferentes áreas de atuação e a apreciação em relação a si mesmo. Esta auto-avaliação permite ao indivíduo orientarse quanto às ações futuras e têm um peso considerável sobre elas. Não se pode deixar de mencionar que parte dessas auto-avaliações é construída a partir de atributos que recebem valoração cultural positiva ou negativa. Elas emergem, ainda, de experiências em que habilidades são postas em jogo e também de contextos sociais que julgam não apenas as habilidades mas os indivíduos como um todo (Bandura 1986). Portanto ter acesso às auto avalia- ções torna-se relevante por duas razões auxiliar na compreensão do indivíduo em si e na identificação das relações que elas mantêm com as ações efetivas. No contexto do estudo da obesidade pode-se pensar em entender como o indivíduo obeso se autoavalia e qual o papel da auto-avaliação, na busca por e na adesão a tratamento.

O presente trabalho propôs-se a identificar o que pensam as crianças obesas sobre seus corpos, ou seja, sua imagem corporal e seu auto-conceito, em relação à aparência física e outros atributos pessoais.

\section{Metodologia}

Participantes - 27 crianças com idades entre 10 e 12 anos, que estavam freqüentando da $4^{\mathrm{a}}$ a $7^{\mathrm{a}}$ série do Ensino Fundamental, em escolas da rede pública da cidade de Ribeirão Preto.

Os critérios de inclusão, no estudo, para estas 27 crianças foram: (1) A criança estar acima do percentil 85 que, segundo critérios National of Health Statistics (NHS) (Must, Dallal \& Dietz, 1991), corresponde a sobrepeso e obesidade; (2) não apresentar problemas orgânicos evidentes e/ou alteracões no desenvolvimento, como retardo neuropsicomotor ou déficits sensoriais. O grupo controle foi constituído também por 27 crianças com idades entre 10 e 12 anos, que estavam cursando as mesmas séries das crianças do grupo de obesos e que estavam em relação ao IMC (Indice de Massa Corporal), situadas entre os percentis 5 e 85 (Must \& cols., 1991).

A caracterização dos participantes é exibida na Tabela 1, abaixo.

\section{Instrumentos utilizados}

EBBIT Eating Behaviors and Body Image Test (Candy \& Fee, 1998), desenvolvido para identificar comportamentos alimentares e imagem corporal em meninas pré adolescentes. O instrumento é um questionário de auto-relato, composto por 42 itens, com 4 alternativas de resposta, no qual cada resposta traduz o comportamento alimentar com uma pontuação diferente. As respostas variam de "a maior parte do tempo" a " nunca” e para cada alternativa escolhida são conferidos pontos de 0 a 3 , sendo: 3 = a maior 
134 Ana Maria Pimenta Carvalho

Tabela 1 - Caracterização dos participantes dos dois grupos.

\begin{tabular}{|c|c|c|c|c|c|c|}
\hline Grupos & Sexo & & Idade & $\mathrm{N}$ & $\begin{array}{l}\text { Percentil } \\
\text { do IMC }\end{array}$ & $\mathrm{N}$ \\
\hline \multirow{4}{*}{$\begin{array}{l}\text { Não obesos } \\
\mathrm{N}=27\end{array}$} & Feminino & 15 & 10 anos & 10 & P 5 & 1 \\
\hline & Masculino & 12 & 11 anos & 8 & P15 & 7 \\
\hline & & & 12 anos & 9 & P50 & 13 \\
\hline & & & & & $50<\mathrm{P}<85$ & 6 \\
\hline Total & & 27 & & 27 & & 27 \\
\hline \multirow{3}{*}{$\begin{array}{l}\text { Obesos } \\
\mathrm{N}=27\end{array}$} & Feminino & 14 & 10 anos & 5 & $85<\mathrm{P}<95$ & 7 \\
\hline & Masculino & 13 & 11 anos & 9 & P 95 & 6 \\
\hline & & & 12 anos & 13 & $\mathrm{P}>95$ & 14 \\
\hline Total & & 27 & & 27 & & 27 \\
\hline
\end{tabular}

parte do tempo; 2 = freqüentemente; 1 = raramente e $0=$ nunca. $O$ teste divide-se em 3 subescalas: insatisfação com a imagem corporal e restrição alimentar, representados por 22 itens; comportamento de comer compulsivo, representado por 15 itens; e uma terceira escala de 4 itens que representa comportamentos compensatórios associados à desordem alimentar indicados no DSM-IV. A primeira e a segunda escala são agrupadas obtendo um escore total e os itens da terceira escala são avaliados individualmente. Este instrumento não tem padronização brasileira. O trabalho de adaptação e validação está em andamento.

Escala Infantil Piers-Harris de Auto Conceito "O que penso e sinto sobre mim mesmo"(Jacob e Loureiro, 1999), originalmente proposta por Piers e Harris (Piers, 1984), foi utilizada na forma traduzida e adaptada por Jacob e Loureiro (1999). Esta escala não tem ainda padronização brasileira. Ela é composta por 80 frases para as quais a criança deve assinalar sim ou não. Este instrumento abrange os seguintes fatores: comportamento; status intelectual e acadêmico; aparência física e atributos; ansiedade; popularidade; e satisfação e felicidade.

\section{Procedimento}

\section{Coleta dos dados}

Os instrumentos foram aplicados na própria escola da criança, após a escola e seus pais terem consentido em sua participação no estudo, em dois momentos diferentes.

O projeto de pesquisa, ao qual este trabalho se vincula, foi aprovado pelo Comitê de Ética em Pesquisa com Seres Humanos da Escola de Enfermagem de Ribeirão Preto, em agosto de 2001.

A duração das aplicações foi de aproximadamente 20 minutos cada uma.

\section{Análise dos dados}

Para análise dos resultados, no presente trabalho, foram utilizadas apenas as sub-escalas relativas a Aparência Física e Atributos, da Escala Infantil Piers-Harris de Auto Conceito e os escores globais do EBBIT e, em seguida, os ítens que avaliam a imagem corporal. Este recorte na avaliação do autoconceito deveu-se a, em trabalho anterior, as questões terem se mostrado como diferenciadoras de obe- 
sos e não obesos e a análise global não ter identificado diferenças entre os grupos (Cataneo, Carvalho \& Galindo, no prelo).

Visto que os instrumentos utilizados não têm normas de padronização para crianças brasileiras, os resultados dos três grupos foram comparados utilizando-se os escores brutos das sub-escalas dos instrumentos de Auto-conceito (Aparência Física e Atributos) e, no caso do EBBIT inicialmente procedeu-se à comparação dos resultados gerais, utilizando-se também os escores brutos e, posteriormente analisou-se apenas as questões relacionadas à percepção da imagem corporal, juntando-se para contagem do número de respondentes, as alternativas "nunca" mais "raramente" e "freqüentemente" mais "a maior parte do tempo".
Para verificação da significância das possíveis diferenças, entre os grupos, nas respostas ao EBBIT, foi utilizado o Teste U de Mann-Whitney (Siegel, 1975).

\section{Resultados}

Neste trabalho serão apresentados apenas os resultados descritivos em termos de freqüência e proporção de respostas às questões dos fatores que tenderam a diferenciar os dois grupos, na Escala de Auto Conceito.

Os resultados em relação às questões da sub escala Aparência Física e Atributos, do instrumento de medida do auto conceito, são apresentadas na Tabelas 2.

Tabela 2 - Freqüência e proporção de respostas sim às afirmações relativas à sub escala Aparência Física e Atributos, da Escala de Auto conceito de Piers Harris, dos dois grupos.

\begin{tabular}{|c|c|c|c|c|}
\hline \multirow[t]{2}{*}{ QUESTÃO } & \multicolumn{2}{|c|}{ Obesos } & \multicolumn{2}{|c|}{ Não obesos } \\
\hline & $\mathbf{F}$ & $\%$ & $\mathbf{F}$ & $\%$ \\
\hline 5. EU SOU ESPERTO & 21 & 81 & 24 & 89 \\
\hline 8. MINHA APARÊNCIA ME INCOMODA & 15 & 58 & 9 & 23 \\
\hline 15. EU SOU FORTE & 21 & 81 & 19 & 70 \\
\hline 29. EU TENHO OLHOS BONITOS & 20 & 77 & 17 & 63 \\
\hline $\begin{array}{l}\text { 33. MEUS AMIGOS GOSTAM DAS MINHAS } \\
\text { IDÉIAS }\end{array}$ & 20 & 77 & 16 & 59 \\
\hline $\begin{array}{l}\text { 42. MUITAS VEZES EU ME OFEREÇO COMO } \\
\text { VOLUNTÁRIO NA ESCOLA }\end{array}$ & 17 & 65 & 16 & 59 \\
\hline $\begin{array}{l}\text { 49. MEUS COLEGAS DE ESCOLA ACHAM QUE } \\
\text { EU TENHO BOAS IDÉIAS }\end{array}$ & 20 & 77 & 16 & 59 \\
\hline 54. EU SOU BONITO & 14 & 54 & 17 & 63 \\
\hline 57. EU SOU POPULAR COM OS MENINOS & 13 & 50 & 13 & 48 \\
\hline 60. EU TENHO UM ROSTO AGRADÁVEL & 18 & 69 & 17 & 63 \\
\hline $\begin{array}{l}\text { 63. EU SOU UM LÍDER EM BRINCADEIRAS E } \\
\text { ESPORTES }\end{array}$ & 9 & 35 & 15 & 55 \\
\hline 69. EU SOU POPULAR COM AS MENINAS & 11 & 42 & 15 & 55 \\
\hline 73. EU TENHO UM CORPO BONITO & 8 & 31 & 15 & 55 \\
\hline
\end{tabular}




\section{Ana Maria Pimenta Carvalho}

Verifica-se que, nas afirmações sobre a aparência e apreciação sobre o próprio corpo, a maior parte das crianças obesas tendeu a concordar com as afirmações sobre o incômodo com sua aparência física, $58 \%$ assinalaram "sim" como resposta e 31\% concordaram que têm um corpo bonito. Em outras questões houve um equilíbrio entre as respostas de crianças obesas e não obesas, sugerindo para as primeiras alguma satisfação consigo mesmas. Por exemplo, elas pensam que têm olhos bonitos e que seus colegas apreciam suas idéias Entre as crianças não obesas, embora percebendo-se como bonitas e concordando menos com a afirmação "minha aparência me incomoda", 55\% consideram que têm um corpo bonito.

Em relação aos escores globais do EBBIT, os valores descritivos são apresentados na Tabela 3.

Tabela 3 - Valores médios dos escores globais, dos dois grupos, no EBBIT.

\begin{tabular}{ccccc}
\hline Grupos & Média & Desvio padrão & Mediana & Amplitude de variação \\
\hline Obesos & 40,54 & 12,05 & 38,5 & $24-70$ \\
Não obesos & 28,95 & 16,90 & 24,5 & $5-59$ \\
\hline
\end{tabular}

A análise comparativa entre os grupos mostrou que eles diferem significativamente. As crianças obesas mostraram comportamentos alimentares indicativos de comer excessivo e insatisfação com a imagem corporal. $(\mathrm{p}=0,05)$

A análise das questões específicas relacionadas à imagem corporal mostrou que as crianças obesas tenderam a concentrar suas respostas nos extremos de concordância com as asserções que vão em direção à insatisfação com o corpo. A Tabela 4 exibe os resultados.

Para as crianças não obesas o contrário ocorre e isto é bem marcante na questão 12 . Na comparação com as crianças obesas, as não obesas colocaram-se majoritariamente contrárias à afirmação. As obesas, por seu turno, distribuíram-se quase que igualmente nos dois extremos, embora tendessem a concordar mais com as alternativas "freqüentemente" e " a maior parte do tempo" achando que são mais gordas que a maioria dos meninos e meninas de mesma idade e altura e que gostariam de que aquela gordura não existisse.

\section{Discussão}

O objetivo do trabalho era identificar o que pensam as crianças obesas sobre seus corpos, ou seja, sua imagem corporal e seu auto conceito, em relação aos atributos físicos e outras características pessoais.

Pode-se verificar que as crianças obesas apresentam um conceito de seus corpos que não é total- mente negativo. Embora estejam majoritariamente incomodados com sua aparência, tendem a concordar com afirmações positivas sobre certas características como ter olhos bonitos, ter um rosto agradável e ser bonito. Mesmo outras características como: julgar que os companheiros apreciam suas idéias, participar em atividades, voluntariamente, na escola, aparecem como fazendo parte do auto-conceito da maioria das crianças obesas, desta amostra. Julgouse bastante relevante avaliar esses outros aspectos que podem estar fazendo o contraponto com as insatisfações ajudando a criança a estruturar-se e até minimizar suas preocupações com a obesidade. Complementarmente, o acesso a esses auto julgamentos auxilia os profissionais que atendem estas crianças a compreendê-las buscando recursos que possam melhor auxiliá-las no processo de adesão a um programa de intervenção.

Os trabalhos revistos na introdução avaliam a representação que a criança faz de outras com diferentes constituições físicas. Seus achados mostram uma representação com conotações negativas em relação a indivíduos com excesso de peso. No presente trabalho buscou-se, como já dito, focalizar as auto-percepções.

A literatura sobre o desenvolvimento aponta que na adolescência há uma tendência ao rebaixamento nas auto-avaliações (Harter, 1990). Em geral, parece que o pré adolescente e o adolescente tornam-se mais críticos em relação a si mesmos, e em 
Tabela 4 - Freqüência e proporção de ocorrência de respostas às questões relativas aos ítens que focalizam a imagem corporal do EBBIT, das crianças dos dois grupos.

\begin{tabular}{|c|c|c|c|c|c|c|c|c|}
\hline \multirow[t]{2}{*}{ Questão } & \multicolumn{2}{|c|}{$\begin{array}{l}\text { Obesos* } \\
\text { Nunca + } \\
\text { raramente }\end{array}$} & $\begin{array}{l}\text { Fr } \\
+ \text { a } \\
\text { do }\end{array}$ & $\begin{array}{l}\text { nente } \\
\text { arte }\end{array}$ & $\begin{array}{l}\text { Nã } \\
\text { Nu } \\
\text { rar }\end{array}$ & & $\begin{array}{l}\text { Fre } \\
+\mathbf{a} \\
\text { do }\end{array}$ & $\begin{array}{l}\text { emente } \\
\text { parte }\end{array}$ \\
\hline & $\mathbf{F}$ & $\%$ & $\mathbf{F}$ & $\%$ & $\begin{array}{l}\mathbf{F} \\
\%\end{array}$ & & $\mathbf{F}$ & $\%$ \\
\hline $\begin{array}{l}\text { 2. meu peso } \\
\text { atual me } \\
\text { incomoda }\end{array}$ & 11 & 42 & 15 & 58 & 20 & 74 & 7 & 26 \\
\hline $\begin{array}{l}\text { 5.eu gostaria de } \\
\text { ser mais } \\
\text { magro(a) }\end{array}$ & 5 & 19 & 21 & 81 & 17 & 63 & 10 & 37 \\
\hline $\begin{array}{l}\text { 10.eu acho que } \\
\text { sou gordo(a) }\end{array}$ & 4 & 15 & 22 & 85 & 19 & 70 & 8 & 30 \\
\hline $\begin{array}{l}\text { 12.eu acho que } \\
\text { sou mais } \\
\text { gordo(a) que a } \\
\text { maioria dos } \\
\text { meninos da } \\
\text { minha idade e } \\
\text { altura }\end{array}$ & 12 & 46 & 14 & 54 & 25 & 93 & 2 & 7 \\
\hline $\begin{array}{l}15 . e u \text { me } \\
\text { preocupo a } \\
\text { respeito de } \\
\text { ganhar peso }\end{array}$ & 7 & 27 & 19 & 73 & 17 & 63 & 10 & 37 \\
\hline $\begin{array}{l}\text { 24.eu me sinto } \\
\text { gordo(a) }\end{array}$ & 3 & 12 & 23 & 88 & 22 & 81 & 5 & 19 \\
\hline $\begin{array}{l}\text { 33.eu olho para } \\
\text { minha gordura } \\
\text { e desejaria que } \\
\text { ela não } \\
\text { existisse }\end{array}$ & 4 & 15 & 22 & 85 & 17 & 63 & 10 & 37 \\
\hline
\end{tabular}

* O total de respondentes é 26 porque um protocolo foi invalidado. 


\section{Ana Maria Pimenta Carvalho}

especial com relação a seus corpos. No presente estudo entre os não obesos há quase um equilíbrio entre concordar e discordar da afirmação: "eu tenho um corpo bonito”. Embora aqui não se tenha feito uma análise por gênero, o resultado é parecido com aquele obtido por Paxton e cols. (1991), mostrando que, também na faixa etária aqui focalizada, há uma preocupação com o corpo e a tendência a que os mais magros estejam mais satisfeitos.

Quanto às respostas ao EBBIT verificou-se que o instrumento parece manter a sensibilidade verificada no estudo de Candy e Fee (1998) ao estabelecer relações entre o IMC e insatisfação com o corpo. No presente trabalho, as crianças obesas tenderam a concentrar suas respostas nos extremos indicativos de insatisfação com seus corpos e sua aparência.

Quanto ao auto conceito, embora não se tenham encontrado resultados globais diferenciando os dois grupos de crianças obesas e não obesas (Cataneo, Carvalho \& Galindo, no prelo), a despeito de outros estudos apontarem nesta direção, ao analisar as asserções relativas à aparência física, duas delas diferenciam os grupos.

Para as afirmações "minha aparência me incomoda”, a maior parte das crianças obesas respondeu concordando e "tenho um corpo bonito", menos da metade da amostra concordou. Contudo há que salientar que nesta questão os não obesos mostraram quase um equilíbrio entre discordar e concordar. Isto sugere uma tendência à insatisfação com o próprio corpo, entre eles. Embora não submetido à verificação pode-se especular que isto se deva ao fato de, neste grupo, haver crianças com índices de massa fronteiriços ao critério de sobrepeso e/ou à tendência de valorização de corpos mais esbeltos, que é um traço cultural marcante, no presente.

A presente análise, incluindo o apoio do instrumento EBBIT possibilitou, a identificação de aspectos diferenciadores de indivíduos obesos e não obesos.

Em uma proposta de trabalho que visa utilizar instrumentos que possam padronizar a forma de coletar as informações e percepções dos sujeitos sobre sua condição, pode-se verificar a sensibilidade dos instrumentos desde que, para a avaliação do autoconceito, se busque uma análise específica das questões que abordam as percepções sobre o corpo. Quan- to ao EBBIT, é necessário reiterar que o instrumento não foi padronizado para a população brasileira, mas parece que se pode verificar uma boa sensibilidade para indicação de preocupações com o excesso de peso. Obesos e não obesos tenderam a responder diferentemente.

Como próximo passo sugere-se buscar entender as relações entre as auto-percepções e o envolvimento com mudanças nos hábitos alimentares buscando reduzir o peso corporal. É preciso conhecer o papel que elas têm sobre o engajamento em programas de tratamento para obesidade.

\section{Referências Bibliográficas}

Azevedo, M. A. (1996). Um estudo exploratório da personalidade da criança obesa através do desenho da figura humana e dos indicadores emocionais de Koppitz, Tese de Doutorado, Faculdade de Ciências Médicas, UNICAMP, Campinas.

Bandura, A. (1986). Social foundations of thought and action. Englewood Cliffs: Prentice-Hall.

Braet, C., Mervielde, I. \& Vandereycken, W. (1997). Psychological aspects of childhood obesity: a controlled study in a clinical and nonclinical sample, Journal of Pediatric Psychology 22 (1), 59-71.

Candy, C. M., \& Fee, V. E. (1998). Underlying Dimensions and Psychometric Properties of the Eating Behaviors and Body Image Test for Preadolescent Girls. Journal of Clinical Child Psychology, 27 (1), 117-127.

Cataneo, C., Carvalho, A M. P. \& Galindo, E. M. C. (no prelo). Obesidade e aspectos psicológicos: maturidade emocional, locus de controle, ansiedade e auto-conceito. Psicologia: Reflexão e Crítica.

Dechen, S., Cano, M.A.T. \& Ribeiro, R.P.P. (2000). A obesidade na adolescência e seus reflexos na auto-imagem corporal. [Resumo] Em Universidade de São Paulo (Org.) CD-Rom $8^{\circ}$ Simpósio Internacional de Iniciação Científica da USP, Ribeirão Preto: USP. 
Galindo, E. M. C., Carvalho, A. M. P., Bugliani, M. A. P., Netto, J. R. da C., Minto, E. C., Dacanal, J. N. \& Montalvão, T. A. G. (2002). Imagem Corporal e o Auto conceito de pré adolescentes obesos. Resumos de Comunicação Científica da XXXII Reunião Anual de Psicologia, (p.357358). Florianópolis: SBP.

Harter, S. (1990). Processes underlying adolescent self-concept formation Em R. Montemayor, G. R. Adams e T. P. Gulotta (Orgs.). From childhood to adolescence: a transitional period? (pp 205-239). Newbury Park: Sage.

Jacob, A .V. \& Loureiro, S. R. (1999). Auto-conceito e desempenho escolar. Resumos de comunicações científicas da XXIX Reunião Anual de Psicologia, (p.164-165), Campinas: SBP.

Kirkpatrick, S. W. \& Sanders, D. M. (1978). Body image stereotypes: a developmental comparison. The Journal of Genetic Psychology, 132, 87-95.

Lawrence, C. M. (1991). Dieting, body image and self concept: their relationship in young children. Master thesis, University of Missouri: Columbia.

Lerner, R. M. \& Korn, S. J. (1972). The development of body build stereotypes in males, Child Development, 43, 908-920.

Must, A ., Dallal, G. E., \& Dietz, W. H. (1991). Reference data for obesity 85th and 95th percentiles of Body Mass Index - a correction. American Journal Clinical Nutrition, 54, 773.

Paxton, S. J., Wertheim, E. H., Gibbons, K., Szmukler, G. I., Hillier, L. \& Petrovich, J. L. (1991). Body image satisfaction, dieting beliefs, and weight loss behaviors in adolescent girls and boys. Journal of Youth and Adolescence, 20, 161-379.

Piers, EV. (1984). Piers-Harris Children's SelfConcept Scale- revised manual Los Angeles: Counselor Psychological Tests, WPS.

Siegel, S. (1975). Estatística não paramétrica. São Paulo: MacGraw Hill.

Stager, S. F. \& Burke, P. (1982). A reexamination of body build stereotypes, Journal of Research in Personality, 16, 435-446.
Thelen, M. H., Powell, A. L., Lawrence, C. \& Kuhnert, M. E. (1992). Eating and body image concerns among children. Journal of Clinical Child Psychology, 21, 41-46.

Este trabalho teve apoio financeiro da FAPESP, com bolsas de Iniciação Científica para Caroline Cataneo e Carolina Tomain Malfará. 